\title{
Two-dimensional resolution of minority carrier diffusion constants in different silicon materials
}

\author{
D. Sontag*, G. Hahn, P. Geiger, P. Fath, E. Bucher \\ Universität Konstanz, Fachbereich Physik, Fach X916, 78457 Konstanz, Germany
}

\begin{abstract}
Hall measurements are a common method to determine the majority charge carrier diffusion constant. But the diffusion constant of the minority carriers $D_{n}$, the more interesting parameter in photovoltaics, is rather hard to detect. In this paper we introduce a method to determine $D_{n}$ locally resolved and mapped in two dimensions. For that purpose the local diffusion length $L_{\text {diff }}$, which can be calculated from LBIC (laser beam induced current) measurements, has been combined with the local bulk lifetime $\tau_{\mathrm{b}}$ received by $\mu$-PCD (microwave-detected photo conductance decay) measurements. We evaluated the diffusion constants of the minority charge carriers $D_{n}$ for different p-type silicon materials with a resolution of $100 \mu \mathrm{m}$. The measurements were carried out on solar cells before and after remote plasma hydrogen passivation in order to get an impression of the diffusion constant dependency on hydrogen incorporation. (C) 2002 Elsevier Science B.V. All rights reserved.
\end{abstract}

Keywords: Minority carrier diffusion constant $D$; Diffusion length $L$; Lifetime $\tau$; Remote plasma hydrogen passivation

\section{Introduction}

Generally, the diffusion constants of silicon materials are considered as constant over the whole volume of a solar cell. Integrating methods like Hall measurements normally deliver the majority carrier diffusion constant averaged over the size of a sample. More difficult is the determination of the diffusion constant for minority carriers, the parameter which is actually more interesting, as minority charge carriers are responsible for the current in a solar cell. There exists a method to determine $D_{n}$ but only for materials with very high lifetimes [1]. In this paper, we would like to

\footnotetext{
*Corresponding author. Tel.: +49-7531-882132; fax: + 49-7531-883895.

E-mail address: detlef.sontag@uni-konstanz.de (D. Sontag).
} 
introduce a method to overcome this problem and to determine $D_{n}$ in different p-type materials locally resolved and mapped in two dimensions. For this reason the diffusion length $L_{\text {diff }}$ and the lifetime $\tau_{\mathrm{b}}$ had to be determined to reveal $D_{n}$ by combining these two parameters.

\section{Experimental approach}

\subsection{Determination of $L_{\mathrm{diff}}$}

The effective diffusion length $L_{\text {eff }}$ can be extracted from an LBIC measurement. Four laser beams with different wavelengths $(635,835,910$ and $980 \mathrm{~nm})$ are generating charge carriers in the bulk of a solar cell [2]. The resulting short circuit currents $I_{\mathrm{SC}}$ as well as the reflection are detected and mapped in two dimensions. Although the maximum resolution of this method is in the range of $5 \mu \mathrm{m}$, we adapted the settings on the minimum stepsize of the $\mu$-PCD device (described in Section 2.2) of $100 \mu \mathrm{m}$ and the minimum spotsize of about $1 \mathrm{~mm}$. By adapting the settings an accurate calculation is guaranteed.

Once having measured the short circuit current, the external quantum efficiency $\mathrm{EQE}(\lambda)$ can be calculated by the equation

$$
\operatorname{EQE}(\lambda)=\operatorname{SR}_{\text {ext }}(\lambda) \frac{h c}{e \lambda}
$$

with the external spectral response

$$
\mathrm{SR}_{\mathrm{ext}}=\frac{I_{\mathrm{SC}}(\lambda)}{F(\lambda)},
$$

the Planck constant $h$, the velocity of light $c$, the electron charge $e$, the wavelength $\lambda$ and the illuminating light power $F(\lambda)$. As we are only interested in photons, which really enter the solar cell, we calculate the internal quantum efficiency $\operatorname{IQE}(\lambda)$ by the equation

$$
\mathrm{IQE}(\lambda)=\mathrm{EQE}(\lambda) \frac{1}{1-R(\lambda)}
$$

with the reflectivity $R(\lambda)$. The absorption of light depends on the wavelength: the smaller the wavelength, the bigger the absorption rate. As a consequence, the $\operatorname{IQE}(\lambda)$ provides information about the effective diffusion length $L_{\text {eff }}$ of the minority charge carriers. A high value of the $\operatorname{IQE}(\lambda)$ indicates large diffusion lengths. The corresponding equation proposed by Basore [3] for untextured solar cells in the range of wavelengths between 800 and $1000 \mathrm{~nm}$ is

$$
\frac{1}{\operatorname{IQE}(\lambda)} \approx 1+\frac{1}{\alpha(\lambda) L_{\text {eff }}} \quad \text { or } \quad L_{\text {eff }} \approx \frac{\operatorname{IQE}(\lambda)}{\alpha(\lambda)(1-\operatorname{IQE}(\lambda))}
$$

with the absorption coefficient $\alpha(\lambda)$.

If the cell thickness $d$ is larger than the bulk diffusion length $L_{\text {diff }}$ it can be shown, that $L_{\text {eff }}$ approximates $L_{\text {diff }}$ [4], because under this condition only a few charge 
carriers reach the back surface and recombinate. With a recombination velocity for our back surface of about $4000-5000 \mathrm{~cm} / \mathrm{s}$ this condition is important for the surface to have no influence on $L_{\text {diff }}$.

The resulting $L_{\text {diff }}$ map is shown in Fig. 1a for an RGS (ribbon growth on substrate) silicon solar cell [5]. This material is still in the R\&D phase but nevertheless very interesting because of low wafering costs (like all ribbon materials) and very high production rate (one wafer per second). The diffusion lengths for this material are typically in the range of $20 \mu \mathrm{m}$ [6]. Comparing this value with the cell thickness of over $200 \mu \mathrm{m}$, the assumption $d>L_{\text {diff }}$ is well satisfied and therefore $L_{\text {diff }}$ equals $L_{\text {eff }}$. Regarding the histogram there are obviously regions with rather high diffusion lengths of more than $40 \mu \mathrm{m}$. These regions possibly indicate the presence of current collection channels as proposed by Häßler et al. [7]. In these regions, $L_{\text {diff }}$ seems to be much higher than in other regions, because channels together with the
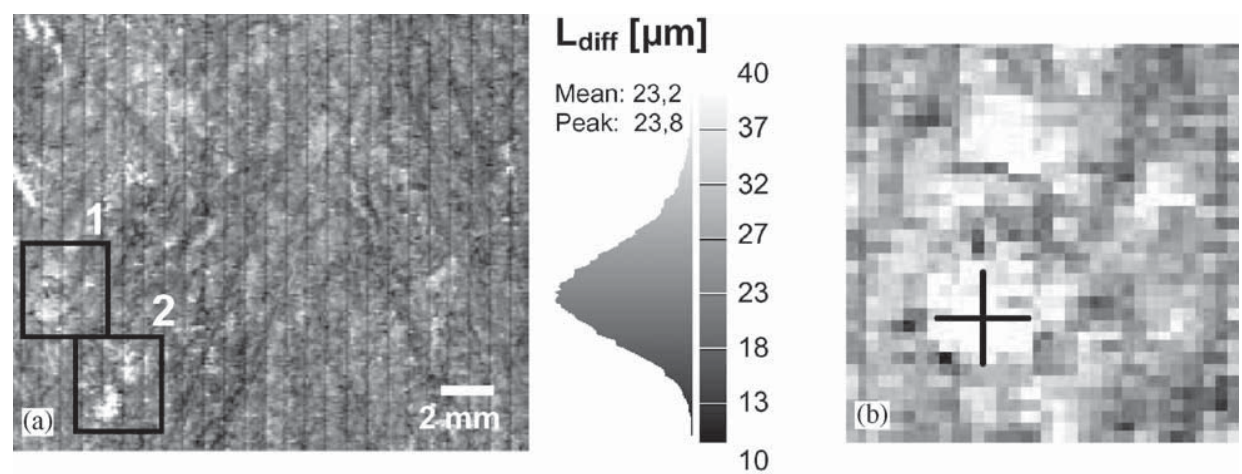

Fig. 1. (a) Map of the diffusion lengths of minority charge carriers in an RGS solar cell. Region 2 indicates an area with no corresponding region in the lifetime map shown below. (b) Enlarged view of region 1 . The cross indicates the selected, distinctive area.
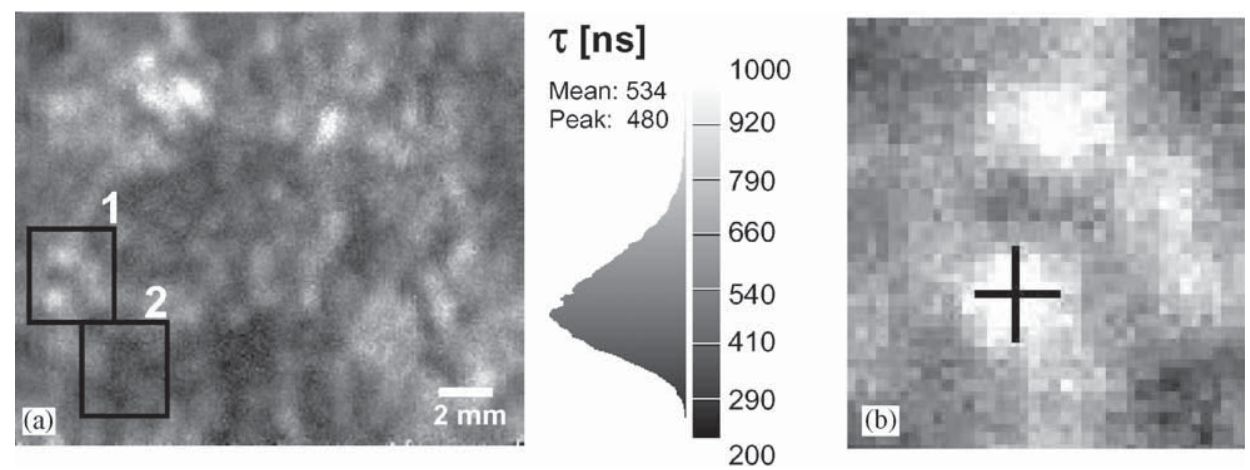

Fig. 2. (a) Map of the lifetime $\tau_{\mathrm{b}}$ of minority charge carriers in the same RGS material as in Fig. 1. There is no comparable, distinctive area to region 2 in Fig. 1a. (b) Enlarged view of region 1. The black cross indicates again the selected region. 
emitter form a three dimensional emitter for which Basore-fit can not be applied. These areas with appearently high diffusion lengths do not automatically correspond to regions with high lifetimes. Comparing Fig. 1a with Fig. 2a there are correlated areas in region 1 but no correlated areas in region 2. Fig. 1b shows an enlarged view of region 1. For the calculation of the diffusion constant described later on it will be important to find the exact position of the small, distinctive area marked with a black cross.

\subsection{Measuring the bulk lifetime $\tau_{b}$}

Another important parameter concerning the performance of solar cells is the bulk lifetime $\tau_{\mathrm{b}}$ of the minority charge carriers. As there are dangling bonds with high recombination activity at the surface of a wafer, always an effective lifetime $\tau_{\text {eff }}$ is measured, depending on the surface recombination velocity $S$, the diffusion constant $D$ and the wafer thickness $d$.

$$
\begin{aligned}
& \frac{1}{\tau_{\mathrm{eff}}}=\frac{1}{\tau_{\mathrm{b}}}+\pi^{2} \frac{D}{d^{2}} \quad \text { for } S \gg \frac{D}{d}, \\
& \frac{1}{\tau_{\mathrm{eff}}}=\frac{1}{\tau_{\mathrm{b}}}+2 \frac{S}{d} \quad \text { for } S \ll \frac{D}{d} .
\end{aligned}
$$

The second equation clarifies that by reducing $S, \tau_{\text {eff }}$ closes up to $\tau_{\mathrm{b}}$. In our experiments we have reached a reduction of $S$ by passivating the surface with iodine/ ethanol. This kind of passivation reduces $S$ to values of $150 \mathrm{~cm} / \mathrm{s}[8]$ and therefore the measured $\tau_{\text {eff }}$ equals $\tau_{\mathrm{b}}$.

The lifetime has been measured with a $\mu$-PCD-device, where a laser beam $(\lambda=905 \mathrm{~nm})$ with a penetration depth of $33 \mu \mathrm{m}$ illuminates a small region generating charge carriers. While these charge carriers recombinate, the conductivity changes exponentially with time. This can be measured by exposing the sample to microwaves and detecting the reflected signal, as microwave reflection is a function of conductivity.

For this examination all lifetime measurements have been performed under low injection conditions. ${ }^{1}$ The frontside grid, the backside contact, and the back surface field had to be removed, as they disturb the measurements and falsify the results. We also removed the emitter in order to perform the measurements with a well-defined surface for passivation. The resulting lifetime map of the same RGS solar cell shown in Fig. 1 is displayed in Fig. 2a. It is remarkable that there are rather big discrepancies in $\tau_{\mathrm{b}}$ between different cell regions. But not all of the regions with high values are visible on both, the $\tau_{\mathrm{b}}$ map and the $L_{\text {diff }}$ map as described in Section 2.1.

For the following calculations we had to choose a region visible on both maps. The selected area, corresponding to Fig. $1 \mathrm{~b}$ for $L_{\text {diff }}$, is displayed in Fig. $2 \mathrm{~b}$ for $\tau_{\mathrm{b}}$ in an enlarged view. Again a black cross marks the position of the selected area as accurately as possible.

\footnotetext{
${ }^{1}$ Low laser intensity.
} 


\section{Evaluating the local diffusion constant $D_{\mathbf{n}}$}

As described above we enlarged a region with a distinctive area in order to find exactly the same position on both, the $\tau_{\mathrm{b}}$ map and the $L_{\text {diff }}$ map. According to these selected points the maps will be combined with respect to the equation

$$
L_{\text {diff }}=\sqrt{D_{\mathrm{n}} \tau_{\mathrm{b}}}
$$

For this purpose, a computer program has been developed to execute this calculation and to display the results. The program enables us to overlay the maps with respect to the position of the selected points. This will combine all the other points of $L_{\text {diff }}$ with the corresponding points of $\tau_{\mathrm{b}}$ as we had chosen the same stepsize for both maps. Otherwise an additional stretching step of one of the maps would have to be carried out. The accuracy in finding exactly the same positions on both maps turned out to be fairly important. A mismatch of two pixels of the two selected points resulted in an error of about 3 percent.

Finally, after taking the square of $L_{\text {diff }}$ and dividing it by $\tau_{\mathrm{b}}$, the resulting $D_{n}$ is shown in Fig. 3.

The reduction of the mean value $\left(12.9 \mathrm{~cm}^{2} / \mathrm{s}\right)$ for RGS of a factor of 2 compared to monocrystalline silicon $\left(27 \mathrm{~cm}^{2} / \mathrm{s}\right)$ [9] is also detected for majority charge carriers, so it can be assumed that in RGS at $300 \mathrm{~K}$ the same scattering mechanisms are responsible for both types of charge carriers.

Regarding $D_{n}$, which is correlated to scattering effects, there is not always a correlation to high $L_{\mathrm{diff}}$ and high $\tau_{\mathrm{b}}$, which indicate areas of low recombination. This means that scattering centers and recombination centers are not necesserily connected.

Furthermore, it is remarkable that the diffusion constant is not homogenous at all, but there are variations of up to a factor of 7 within a solar cell. For this material with its low diffusion lengths it is no problem to satisfy Basore conditions as long as
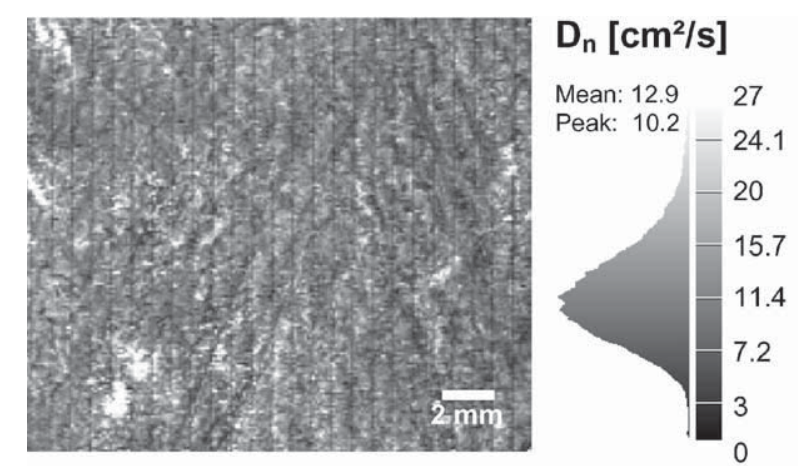

Fig. 3. Map of the minority charge carrier diffusion constant $D_{n}$ of RGS material. The peak value indicates the value measured most frequently. 
Table 1

$D_{n}$ calculated for different types of silicon materials

\begin{tabular}{lll}
\hline Material & $\begin{array}{l}\text { Before H-passivation }\left(\mathrm{cm}^{2} / \mathrm{s}\right) \\
\text { Mean value }\end{array}$ & $\begin{array}{l}\text { After H-passivation }\left(\mathrm{cm}^{2} / \mathrm{s}\right) \\
\text { Mean value }\end{array}$ \\
\hline RGS, ribbon & - & 13 \\
EFG, ribbon & 17 & 14 \\
Baysix, ingot & 22 & 23 \\
\hline
\end{tabular}

For EFG and Baysix to satisfy Basore condition only regions with low diffusion lengths were considered

there are no current collection channels. For other materials like EFG or Baysix it is not that simple as discribed in the next section.

\section{Results}

The method described above for determination of the local diffusion constant of the minority charge carriers was applied to different kinds of multicrystalline silicon materials. Besides of ribbon materials like RGS and EFG (edge-defined film-fed growth from ASE) also Baysix material (from Bayer AG) was used. The calculations were carried out before and after remote plasma hydrogen passivation [10] for EFG and Baysix materials in order to investigate the influence of the hydrogen passivation effect on the diffusion constant. Therefore, after the first LBIC measurements without H-passivation the solar cells were hydrogen passivated and again LBIC measured. Then contacts, back surface fields and emitters were removed. After that the first $\mu$-PCD measurement was performed. Finally, after tempering the samples at $475^{\circ} \mathrm{C}$ for $10 \mathrm{~h}$ to remove the hydrogen, the wafers were $\mu$-PCD measured for the second time. This sequence enabled us to carry out all the needed measurements to determine $D_{n}$. The results are shown in Table 1 . We have to keep in mind that diffusion lengths over about $150 \mu \mathrm{m}$ do not match the conditions for the fit proposed by Basore. So these values as well as lifetimes higher than in monocrystalline silicon were excluded for Baysix and EFG material before the calculations. As a result, for Baysix material mainly regions around grain boundaries and for EFG only crystals with poor diffusion lengths remained for measurement.

Concerning the remote H-passivation there seems to be no effect of the hydrogen on the diffusion constants in this first experiment. For all materials the mean values do not change significantly. In EFG material the tendency is even to lower values.

\section{Summary}

The method introduced in this paper is a possible way to reveal the local diffusion constant of the minority charge carriers in two dimensions for silicon materials with low diffusion lengths like RGS, but in areas with high $L_{\text {diff, }}$ indicating current 
collecting channels, Basore-fit cannot be applied because in these regions the emitter has a three dimensional structure. For other materials like Baysix and EFG higher values for $L_{\text {diff }}$ than the cell thickness $d$ have to be neglected in order to satisfy Basore condition. Until now there is no visible tendency concerning the influence of hydrogen on the diffusion constant. Further measurements also with other materials like string ribbon (from evergreen solar) will be carried out to improve statistics.

\section{Acknowledgements}

This work was supported within the KoSi program by the German Bundesministerium für Wirtschaft (BMWi) under contract number 0329858J.

We gratefully acknowledge the computational support of T. Pernau, and we would also like to thank M. Keil for technical assistance during cell processing.

\section{References}

[1] A.B. Sproul, M.A. Green, A.W. Stephens, J. Appl. Phys. 72 (9) (1992) 4161.

[2] T. Pernau, Lebensdauerbestimmung und ortsaufgelöste Messung der Quantenausbeute an kristallinem Silizium, Diplomarbeit, Universität Konstanz, 1999, http://www.ub.uni-konstanz.de/ kops/volltexte/1999/304/.

[3] P.A. Basore, IEEE Trans. Electron Devices ED-37 (1990) 337.

[4] W. Jooss, Getterstudien an multikristallinem Silizium für Solarzellen, Diplomarbeit, Universität Konstanz, 1998.

[5] H. Lange, I.A. Schwirtlich, J. Crys. Growth 104 (1990) 108.

[6] G. Hahn, C. Zechner, M. Rinio, P. Fath, G. Willeke, E. Bucher, J. Appl. Phys. 86 (12) (1999) 7179.

[7] C. Häßler, H.-U. Höfs, S. Thurm, Proceedings of the Second WCPVSEC, Vienna, 1998, pp. 1886-1889.

[8] J.A. Eikelboom, C. Leguijt, A.R. Burgers, Proceedings of the 12th EC PVSEC, Amsterdam, 1994, p. 1782.

[9] A.W. Stephens, M.A. Green, J. Appl. Phys. 74 (10) (1993) 6212.

[10] M. Spiegel, et al., Proceedings of the 13th EC PVSEC, Nizza, 1995, p. 421. 